\title{
Tum1 is involved in the metabolism of sterol esters in Saccharomyces cerevisiae
}

Katja Uršič ${ }^{1,4}$, Mojca Ogrizović ${ }^{1}$ Dušan Kordiš ${ }^{1}$, Klaus Natter $^{2}$ and Uroš Petrovič ${ }^{1,3^{*}}$ (D)

\begin{abstract}
Background: The only hitherto known biological role of yeast Saccharomyces cerevisiae Tum 1 protein is in the tRNA thiolation pathway. The mammalian homologue of the yeast TUM1 gene, the thiosulfate sulfurtransferase (a.k.a. rhodanese) Tst, has been proposed as an obesity-resistance and antidiabetic gene. To assess the role of Tum1 in cell metabolism and the putative functional connection between lipid metabolism and tRNA modification, we analysed evolutionary conservation of the rhodanese protein superfamily, investigated the role of Tum 1 in lipid metabolism, and examined the phenotype of yeast strains expressing the mouse homologue of Tum1, TST.

Results: We analysed evolutionary relationships in the rhodanese superfamily and established that its members are widespread in bacteria, archaea and in all major eukaryotic groups. We found that the amount of sterol esters was significantly higher in the deletion strain tum $1 \Delta$ than in the wild-type strain. Expression of the mouse TST protein in the deletion strain did not rescue this phenotype. Moreover, although Tum1 deficiency in the thiolation pathway was complemented by re-introducing TUM1, it was not complemented by the introduction of the mouse homologue Tst. We further showed that the tRNA thiolation pathway is not involved in the regulation of sterol ester content in S. cerevisiae, as overexpression of the $\mathrm{tE}^{\mathrm{UUC}}, \mathrm{tK}^{\mathrm{UUU}}$ and $\mathrm{tQ}^{\mathrm{UUG}}$ tRNAs did not rescue the lipid phenotype in the tum $1 \Delta$ deletion strain, and, additionally, deletion of the key gene for the tRNA thiolation pathway, UBA4, did not affect sterol ester content.

Conclusions: The rhodanese superfamily of proteins is widespread in all organisms, and yeast TUM1 is a bona fide orthologue of mammalian Tst thiosulfate sulfurtransferase gene. However, the mouse TST protein cannot functionally replace yeast Tum1 protein, neither in its lipid metabolism-related function, nor in the tRNA thiolation pathway. We show here that Tum 1 protein is involved in lipid metabolism by decreasing the sterol ester content in yeast cells, and that this function of Tum1 is not exerted through the tRNA thiolation pathway, but through another, currently unknown pathway.
\end{abstract}

Keywords: Lipid metabolism, tRNA modification, Yeast Saccharomyces cerevisiae

\section{Background}

Lipids are important dynamic molecules that play vital roles, which extend beyond their structural roles in cellular membranes [1-4]. Lipid metabolism has lately gained much attention in particular due to its role in health and disease, especially in the emerging pandemics of obesity and type 2 diabetes [5, 6]. Recently, mouse thiosulfate sulfurtransferase (TST) protein was proposed

\footnotetext{
* Correspondence: uros.petrovic@bf.uni-lj.si

${ }^{1}$ Jožef Stefan Institute, Department of Molecular and Biomedical Sciences, Jamova cesta 39, 1000 Ljubljana, Slovenia

${ }^{3}$ Biotechnical Faculty, Department of Biology, University of Ljubljana, Večna pot 111, 1000 Ljubljana, Slovenia

Full list of author information is available at the end of the article
}

to have obesity-resistance and anti-diabetes effects in mammals [7]. The mechanism of action causing the decreased accumulation of storage lipids was proposed to be increased expression and activity of the protein, resulting in augmented mitochondrial function and increased degradation of reactive oxygen species and sulfide.

TUM1 (YOR251c) is a unique yeast Saccharomyces cerevisiae ortholog of the human TST gene [8]. Tum1 and TST/MPST (mercaptopyruvate sulfurtransferase) proteins belong to the rhodanese/cell cycle control phosphatase protein superfamily. The rhodanese superfamily contains the fold with the same name and the proteins from this superfamily span a wide range of molecular 
functions. In this study we examined the molecular role of the yeast Tum 1 protein which has been previously described only as a protein with a non-essential function in the tRNA wobble uridine modification pathway $[9,10]$. This pathway consists also of Uba4, Ncs2, Ncs6 and Nfs1 proteins and is required for the thiolation of three tRNA molecules, specific for glutamine $\left(\mathrm{tQ}^{\mathrm{UUG}}\right)$, lysine $\left(\mathrm{tK}^{\mathrm{UUU}}\right)$, and glutamic acid ( $\mathrm{E}^{\mathrm{UUC}}$ ) tRNAs, which contain 2-thiouridine derivate as the wobble nucleoside [11]. To date, 156 genes have been annotated to lipid metabolism in S. cerevisiae [12] and additional proteins are still being described as involved in this complex process [4]. However, no functional connection between tRNA modification and lipid metabolism has been established yet, opening a question of structural and functional conservation of the rhodanese superfamily members in general, and Tum1 and TST in particular. We therefore analysed evolutionary conservation of the rhodanese protein superfamily, investigated the role of Tum1 in lipid metabolism, and examined the putative functional connection between lipid metabolism and tRNA modification processes.

\section{Methods}

\section{Sequence data mining}

All database searches were performed online. The databases analysed were the prokaryotic and eukaryotic protein, transcriptome and genome databases at the National Center for Biotechnology Information [13]. To detect all available representatives of the rhodanese superfamily, database searches were performed iteratively. Comparisons were performed using the TBLASTN program [14], with the E-value cutoff set to $10^{-5}$ and default settings for other parameters. Diverse prokaryotic and eukaryotic representatives of the rhodanese superfamily $[15,16]$ have been used as queries. The Translate program from Expasy [17] was used to translate DNA sequences.

\section{Phylogenetic analysis}

Eukaryotic and prokaryotic representatives of the rhodanese superfamily $[15,16]$ were included in the analyses. Diverse opisthokont representatives of the rhodanese superfamily were also included in the separate analysis. The rhodanese domain in the newly discovered representatives of the rhodanese superfamily was identified by using the SMART [18], InterPro [19] and Pfam [20] domain databases. The protein sequences were aligned using Clustal Omega [21]. We used IQ Tree web server [22] to select the best maximum likelihood model of sequence evolution for the data from the rhodanese superfamily. The best fit model of amino acid substitution for our data set was determined as LG + G4 according to BIC (Bayesian information criterion). Phylogenetic trees were reconstructed using the maximum likelihood method [23] and the neighbor-joining method [24]. The reliability of the resulting topologies was tested by the bootstrap method. Two archaeal rhodanese superfamily representatives were used as the outgroup in the global rhodanese phylogeny, while the Arabidopsis TST1 repeat domain of rhodanese protein was used to root the Opisthokonta rhodanese tree. Phylogenetic analyses were performed with IQ Tree web server [22].

\section{Synteny analysis of the rhodanese superfamily in vertebrates}

For synteny analyses, the chromosomal locations, lengths, and the directionality of the neighbouring genes upstream and downstream of Tst and Mpst genes were extracted from Ensembl [25] and Entrez [26] genome databases. Additional synteny comparisons were conducted using Genomicus [27].

\section{Strains and media}

The Saccharomyces cerevisiae wild-type (WT) strain

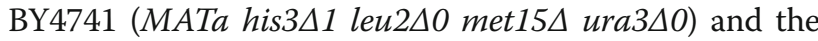
isogenic $u b a 4 \Delta$ deletion strain were obtained from the EUROSCARF strain collection. The tum $1 \Delta$ deletion strain was constructed by replacing the TUM1 open reading frame (ORF) with the $\operatorname{KanMX}$ resistance cassette. A strain with re-inserted TUM1 was used as the control strain. The ORF of the Tst gene was PCRamplified from the appropriate mouse (Mus musculus) cDNA clone and introduced into the TUM1 locus, under the control of the endogenous yeast promoter.

Strains were grown in rich medium YPD ( $2 \%$ peptone, $1 \%$ yeast extract, $2 \%$ glucose) or in synthetic complete medium lacking uracil (SC-Ura). YPD plates containing $0.5 \mathrm{mg} / \mathrm{ml}$ geneticin (G418, Formedium) or $0.1 \mathrm{mg} / \mathrm{ml}$ nourseothricin (clonNAT, Werner) were used to select for geneticin or nourseothricin resistance.

\section{Plasmid construction}

DNA manipulations, plasmid preparations and bacterial transformations were performed according to standard methods. For expression of TUM1 or Tst under control of the $A D H 1$ promoter, the respective ORFs were inserted into the pUG35 plasmid backbone [28] immediately after the $A D H 1$ promoter. Plasmids were named according to inserts: [empty] for backbone only, [TUM1] for the plasmid expressing yeast TUM1 and [Tst] for the plasmid expressing mouse Tst.

Plasmids pABY1653 ( $\left.\mathrm{tE}^{\mathrm{UUC}}-\mathrm{tK}^{\mathrm{UUU}}-\mathrm{tQ}^{\mathrm{UUG}}\right)$ and pABY525 (control), described in [29], were kindly provided by Prof. Anders Byström, Umeå University, Sweden.

\section{Isolation and purification of tRNA}

Yeast strains were grown in $100 \mathrm{ml}$ of YPD or SC-Ura medium and harvested during exponential phase of 
growth $\left(\mathrm{OD}_{600}=0.5-0.6\right)$. Total RNA was isolated using hot acid phenol chloroform extraction. We separated tRNA molecules from other types of RNA molecules as described [30]. The isolated tRNA molecules were further purified by denaturing polyacrylamide gel electrophoresis containing $10 \mu \mathrm{g} / \mathrm{ml} \mathrm{N}$-acryloylaminophenylmercuric chloride (APM). The presence of thiouridine in tRNAs was determined by the decrease of electrophoretic mobility on polyacrylamide gel.

\section{Isolation of proteins, SDS-PAGE and western blot}

Yeast strains were grown in $20 \mathrm{ml}$ of SC-Ura medium and harvested during exponential growth phase $\left(\mathrm{OD}_{600}=0.5-0.6\right)$. Proteins were isolated as described [31]. Mouse liver tissue whole-cell homogenates were made with RIPA buffer (1\% NP-40, $0.1 \%$ SDS, $0.5 \%$ Na-Deoxycholate in PBS) with added protease inhibitors and used directly for western blot analysis. Yeast and mouse protein mixtures were separated in duplicates on a $12.5 \%$ SDS gel with a $4 \%$ stacking gel at $30 \mathrm{~mA}$ and $300 \mathrm{~V}$. The proteins were transferred to a nitrocellulose membrane, which was afterwards blocked with 5\% low fat milk in Tris buffered saline TBS, $0.05 \%$ Tween-20 (TBST) for $1 \mathrm{~h}$, and then incubated overnight at $4{ }^{\circ} \mathrm{C}$ with the anti-TST primary antibody (EPR1164G(B), RabMAb, 1:1000). After washing with TBST, the membrane was incubated with secondary antibodies in 5\% blocking buffer (Goat Anti-rabbit pod, 111-035-003, Jackson Immunoresearch, 1:10,000), and scanned.

\section{Lipid analysis}

Approximately $2 \times 10^{9}$ cells were harvested from exponentially growing cultures (SC medium) and subjected to glass bead disruption in chloroform:methanol 2:1 in a test tube shaker (Heidolph Multi Reax). Lipids were quantitatively extracted with chloroform:methanol 2:1 and analysed by thin layer chromatography (TLC) as described in [32]. For dry weight determination, the cultures were filtrated through $0.45 \mu \mathrm{m}$ cellulose nitrate filters (Sartorius Stedim) and dried overnight at $95{ }^{\circ} \mathrm{C}$.

\section{Statistical analysis}

Data were tested for normality of distribution by Sharpiro-Wilk test. All pair-wise multiple comparisons were tested using the Holm-Sidak method after one-way analysis of variance. A $p$-value of less than 0.05 was considered to be statistically significant. Data are expressed as average \pm standard deviation. SigmaPlot Software (Systat Software Inc) and Excel (Microsoft Office) were used for graphical representation and statistical analysis.

\section{Results}

Evolutionary relationships in the rhodanese superfamily To determine evolutionary relationships in the rhodanese superfamily, of which TUM1 and Tst are members, the data available in sequence databases were analysed to resolve the origin and evolutionary history of the superfamily members. The phyletic distribution pattern of the rhodanese superfamily was first analysed. According to global phylogenetic analysis, based on our collection of the representatives, the rhodanese superfamily is widespread in bacteria, archaea and in all major eukaryotic groups (Fig. 1a). Global phylogenetic analysis failed to obtain any evidence for orthologous groups within the rhodanese superfamily (Fig. 1a) and rhodanese representatives from major eukaryotic supergroups mostly did not group in the supergroup manner. The prokaryotic sequences were scattered among the eukaryotic representatives and did not form a monophyletic clade. Moreover, the levels of sequence identity between some prokaryotic and eukaryotic rhodanese representatives reached up to $50 \%$ at the amino acid level. The neighborjoining method with uncorrected distances was found to produce a similar resolution in the rhodanese superfamily as the more complex maximum likelihood method (data not shown).

In the metazoan and fungi (Opisthokonta) tree the representatives of the rhodanese superfamily are relatively well separated (Fig. 1b). Human TST and MPST show $59 \%$ amino acid identity, while the human TST compared to yeast Tum 1 shows $32 \%$ amino acid identity, and human MPST compared to yeast Tum1 shows $37 \%$ amino acid identity (Fig. 2).

\section{Conserved synteny of the rhodanese superfamily in vertebrates}

Ancestral states of the Tst and Mpst chromosomal positions were reconstructed from comparisons of syntenic positions between the diverse vertebrate lineages Homo, Mus, Gallus, Anolis, Amniota, Silurana and Latimeria. Specifically, the identity and relative genomic position of the genes that map to genomic regions immediately adjacent to either side $\left(5^{\prime}\right.$ vs. $\left.3^{\prime}\right)$ of all vertebrate rhodanese superfamily genes were recorded using the Ensembl and Entrez genome databases as well as the Genomicus database. Analysis of conserved synteny demonstrated that the gene duplication of rhodanese gene superfamily occurred in the ancestor of land vertebrates ( 359 Mya), producing Tst and Mpst genes. Tst and Mpst are duplicated genes, located in the tail to tail orientation (Fig. 3).

\section{Effect of Tum 1 on the lipid content in S. cerevisiae}

Next, we investigated the involvement of TUM1 in lipid metabolism of $S$. cerevisiae. Three different groups of lipids 

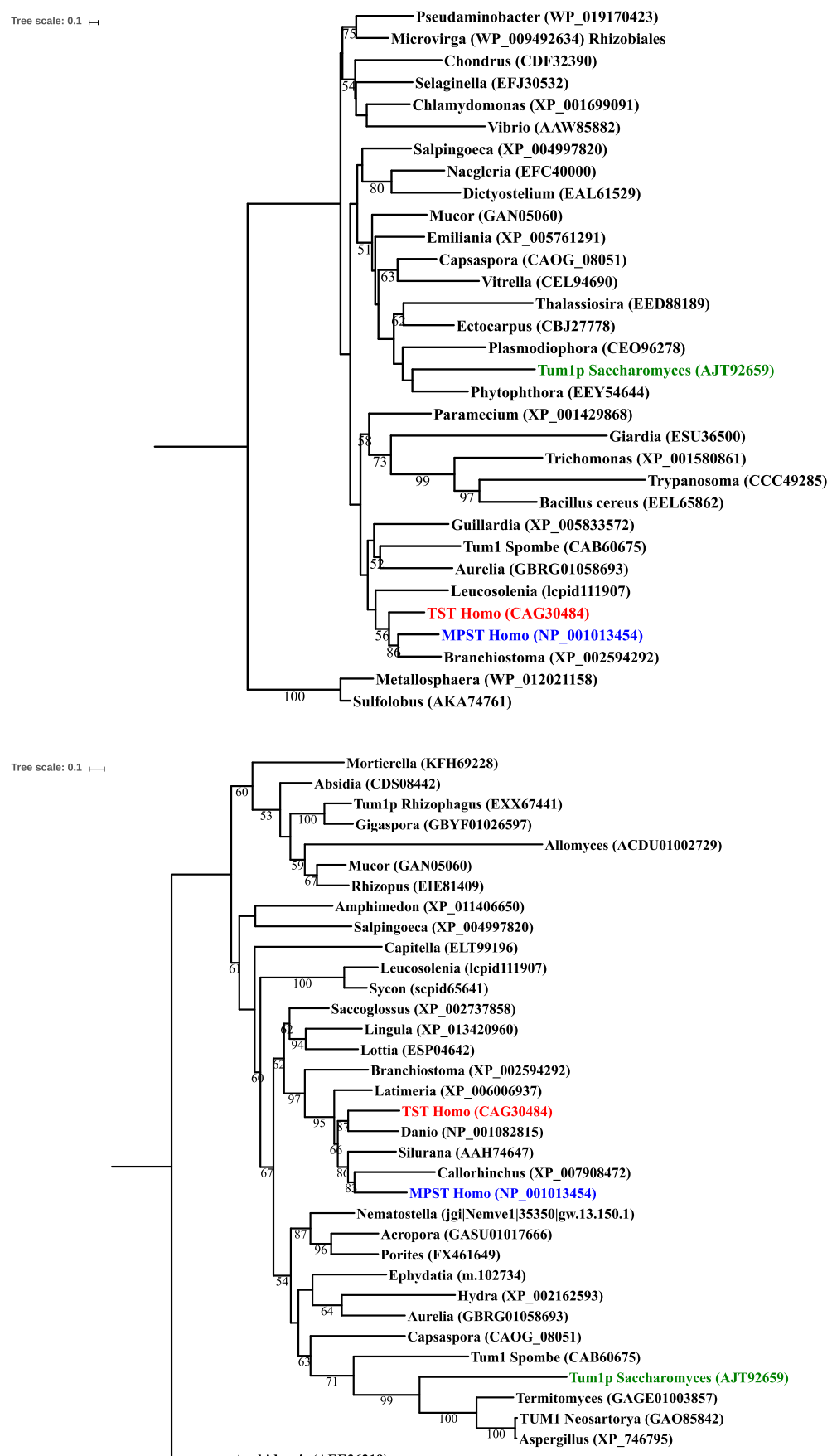

Fig. 1 Phylogeny of the rhodanese superfamily. a Global rhodanese phylogeny. Two archaeal rhodanese representatives were used to root the tree. $\mathbf{b}$ Rhodanese superfamily in Opisthokonta. The TST1 repeat domain of the rhodanese protein from Arabidopsis (AEE36219) was used to root this tree. In both cases, the rooted maximum likelihood tree was inferred by IQ Tree [22] under a LG + G4 model from the TST1 repeat domain, and reliability for the internal branches was assessed using the 1000 bootstrap replications. Nodes with confidence values greater than $50 \%$ are indicated. All sequences were obtained from the GenBank; genus names and accession numbers are included

were measured, by TLC: ergosterol (ERG), triacylglycerols (TAG) and sterol esters (SE). The lipid content was first compared in the WT and the tum1 $1 \Delta$ deletion strains of $S$. cerevisiae. The amount of SE was $60 \%$ higher in the deletion than in the WT strain (Fig. 4a). In contrast, the TAG content did not significantly $(p>0.05)$ differ between the cells of these two strains, and ERG levels were also comparable (Fig. 4a). 


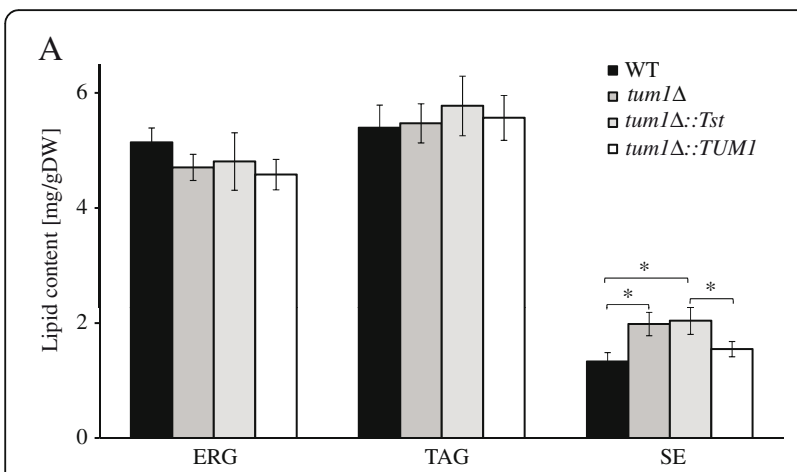

B

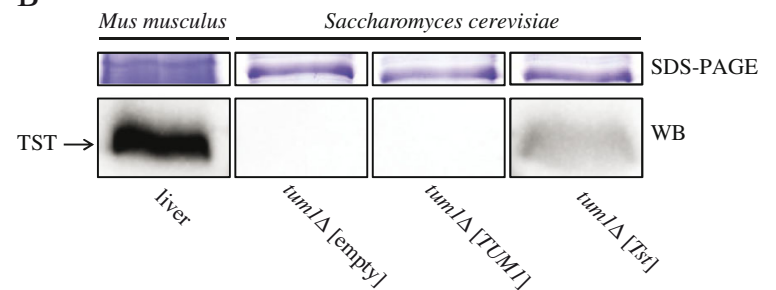

Fig. 4 Yeast Tum 1 and mouse Tst affect lipid metabolism in the Saccharomyces cerevisiae cells. a Lipid analysis of neutral lipids was performed by TLC. Three different groups of lipids were measured: ergosterol (ERG), triacylglycerols (TAG) and sterol esters (SE). The error bars represent standard deviation. Deletion of TUM1 causes a $60 \%$ increase of SE, as compared to the wild-type. This phenotype is also observed in the strain expressing Tst, indicating that Tst is not able to complement the loss of TUM1. b Western blot analysis confirms the presence of Mus musculus TST in the cells of Saccharomyces cerevisiae. Extracts of proteins were prepared and analysed by polyacrylamide gel electrophoresis and by western blotting with anti-TST antibodies. The Mus musculus liver protein sample was used as a positive control and tum14 and tum 14::TUM1 as negative controls. Coomassie Brilliant Blue staining of proteins in the gel with the same amount of protein as for the western blot analysis was used as a loading control. Boxes denote different parts of either the same gel (SDS-PAGE) or membrane (WB)

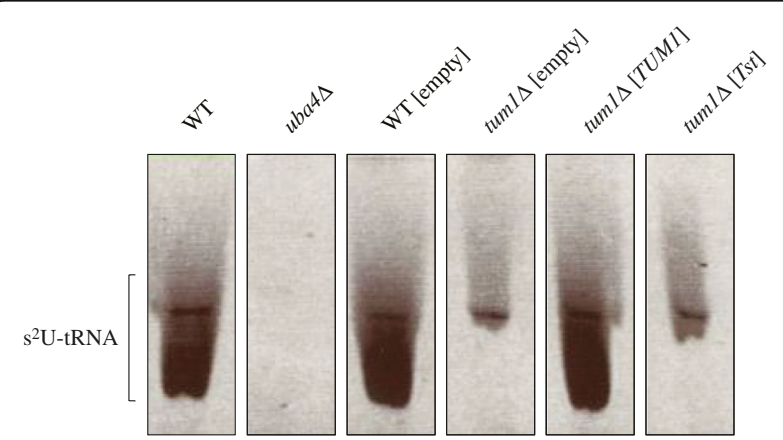

Fig. 5 Mouse TST protein cannot complement Tum1 deficiency in the tRNA thiolation pathway. The tRNA thiolation status was analysed in the wild-type strain (WT), the strain without Uba4 protein (uba44), and in strains transformed with empty plasmids (WT[empty], tum 14[empty]) and with plasmids with inserted yeast and mouse homologues (tum14[TUM1], tum14[Tst]). Total tRNA was resolved by urea polyacrylamide-gel electrophoresis in the presence of APM
Cross-talk between the tRNA thiolation pathway and lipid content in S. cerevisiae

Finally, we investigated the putative connection between storage lipid content and the tRNA thiolation in $S$. cerevisiae. The measurement of the storage lipid content in the uba4 $\Delta$ strain showed that the SE content did not differ from the WT strain (data not shown). To address this putative cross-talk from a different angle, we inserted into the tum1 1 deletion strain the pABY1653 plasmid encoding $\mathrm{tE}^{\mathrm{UUC}}, \mathrm{tK}^{\mathrm{UUU}}$ and $\mathrm{tQ}^{\mathrm{UUG}} \mathrm{tRNAs}$, which has been shown to rescue the phenotype caused by the deletion of the genes involved in the tRNA thiolation pathway [10]. The amount of SE, however, did not differ from the tum $1 \Delta$ deletion strain or from the same strain transformed with an empty (control) plasmid. Additionally, we determined that the SE content in the $u b a 4 \Delta$ deletion strain overexpressing the three tRNAs molecules was the same as in the $u b a 4 \Delta$ deletion control strain.

\section{Discussion}

The purpose of this study was to determine evolutionary relationships in the rhodanese superfamily and to investigate the molecular function of one superfamily member, Tum 1 protein, encoded by the yeast homologue of the mammalian Tst gene. To establish evolutionary relationships between TUM1, Tst, and its paralogue Mpst, the analysis was done both at the global level, including genomes from bacteria, archaea and eukaryotes, and at the level of animals and fungi (Opisthokonta). The protein sequences in the rhodanese superfamily are challenging for a phylogenetic analysis, as they are divergent and produce low bootstrap values in the phylogenetic trees. We found that the reconstruction of evolutionary relationships in the rhodanese superfamily is relatively simple on the short evolutionary timescale (e.g., in Opisthokonta), but it becomes more difficult on the larger evolutionary timescale. In the reconstruction of the evolutionary history in the rhodanese superfamily it was helpful that prokaryotes and eukaryotes contain only a single lineage. The analysis demonstrated that rhodanese superfamily genes are widespread in bacteria, archaea and in all major eukaryotic groups. The prokaryotic sequences do not form a monophyletic clade, but are instead scattered among the eukaryotic representatives, indicating the possibility of horizontal gene transfer, which is further supported by relatively high levels of sequence identity between some prokaryotic and eukaryotic rhodanese representatives.

Tum1 and TST/MPST proteins are representatives of the multidomain sulfurtransferase (rhodanese) superfamily [33]. According to this database, yeast $S$. cerevisiae possesses 11 proteins that belong to the rhodanese superfamily. These proteins belong to three protein families, to 
the "multidomain sulfurtransferase (rhodanese)" (Tum1, Rdl1, Rdl2 and Ubp7), to the "cell cycle control phosphatase, catalytic domain" (Doa4, Ptp3, Ubp5, Ych1, Mih1 and Arr2) and to the "single domain sulfurtransferase" (Uba4). Tum1 and TST possess the same domain organization of two duplicated TST repeat domains, while the other yeast representatives of the multidomain sulfurtransferase (rhodanese) superfamily - Rdl1, Rdl2 and Ubp7 are multidomain proteins with the fused rhodanese domain. In contrast to yeast, humans contain a much larger number of proteins that belong to the rhodanese superfamily, i.e. 71 proteins in total according to the Superfamily database [33].

Synteny analyses can be a powerful tool for establishing gene homology relationships and for providing clues about the mechanisms of origins of new genes, as the order and orientation of genes tend to remain the same over relatively long evolutionary time. We therefore reconstructed the ancestral state of the chromosomal positions of the rhodanese superfamily genes. Analysis of conserved syntenies showed clearly that the gene duplication of the rhodanese superfamily occurred 359 My ago in the ancestor of land vertebrates, producing Tst and Mpst genes.

Having established a direct orthologous relation between TUM1 and Tst genes, we addressed the question of their functional similarity. We found that the amount of SE, but not of TAG or ERG, was significantly higher in the strain lacking TUM1. The increased levels of SE in the mutant strain indicate higher activity of the ergosterol pathway, resulting in storage of excess ERG as SE. Namely, the main role of SE is assumed to be storage of excess ERG, in order to keep the concentration of ERG in the membrane constant. According to this interpretation, Tum1 inhibits the ergosterol biosynthetic pathway. Another explanation is also possible, however: while the difference in the ERG content between the WT strain and the strain lacking TUM1 is not significant, our data indicate that it may be slightly lower in the mutant, meaning that the total ergosterol levels (i.e. ERG + ergosterol in SE) do not differ so considerably between the strains. The difference in distribution of ERG could be a consequence of difference in the cell size, resulting in different membrane surface for the same biomass. Indeed, we observed that cells of the tum1 $\Delta$ strain are approximately $55 \%(p<0.01)$ larger than those of the WT strain, in accordance with the latter interpretation. Still, such redistribution of ERG between membranes and as SE in lipid droplets has not been described for other strains which differ in cell size from the WT, and, based on our data, it can only be assumed that Tum1 has a direct effect on the ergosterol pathway. These results thus indicate that Tum1 is indeed involved in lipid metabolism, specifically in the control of the squalene and/or ergosterol pathway.
To determine whether TUM1 and Tst genes remained functionally complementary over the 1.2 billion to 1.5 billion years of evolution [34], we replaced the yeast nonessential TUM1 gene with the mouse Tst, and assayed for the complementation of altered SE content. However, mouse TST could not complement the function of yeast Tum1 protein in its lipid metabolismrelated function. Focusing on the pathway for 2thiolation of the wobble uridine base of the specific tRNA molecules, which hitherto is the only known biological process in which Tum 1 is involved, a similar result was obtained. Tum1 is not absolutely required for the biosynthesis of 2-thiouridine, contrasting its role to that of Uba4, but its activity is necessary for wild-type levels of 2-thiouridine [9-11]. On the other hand, mouse TST protein has never been implicated in the tRNA thiolation pathway. The level of tRNA thiolation was only partially decreased in the tum $1 \Delta$ deletion strain, whereas it was completely absent in the $u b a 4 \Delta$ strain, in agreement with previous findings $[9,10]$. Tum 1 deficiency in the thiolation pathway was, however, not complemented by the introduction of the mouse homologue Tst. We thus demonstrated that the mouse TST protein cannot functionally replace Tum 1 deficiency in the tRNA thiolation pathway in yeast, too. It should be noted, however, that even when orthologues perform similar functions in different organisms, it may not be possible to replace one for the other, particularly if the organisms are as widely diverged as mouse and yeast [35]. This result therefore cannot be extended to imply the molecular function of Tst in mammals.

Are the roles of Tum1 in lipid metabolism and in tRNA modification directly linked? We found no evidence of the effect of deletion of $U B A 4$ on lipid content. Since Uba4 is downstream from Tum1 in the tRNA thiolation pathway $[9,10]$, this strongly indicates that tRNA thiolation and storage lipid content pathways are not connected. In addition, over-expression of tRNAs that can rescue the phenotype caused by the deletion of the genes involved in the tRNA thiolation pathway, did not rescue or suppress the lipid phenotype of the tum $1 \Delta$ deletion strain, nor did it alter the lipid content in the uba4s deletion strain. These results strongly support the conclusion that the tRNA thiolation pathway is not involved in the regulation of SE content in S. cerevisiae. Notably, the existence of a function for Tum1 in an additional, yet currently unknown pathway has been proposed previously [10].

\section{Conclusion}

The aim of functional genomics has been to determine the function of all the genes comprising a given genome. As several well-studied examples have shown, the property of executing more than one molecular function is far from 
exceptional. In light of this, determining all the functions for a given gene/protein will most likely be one of the next challenges in functional genomics. The importance of this challenge is further exacerbated when the function of orthologous genes is supported or predicted based on the known function of only one from the pair of the orthologues. Moreover, interactions between cellular pathways or processes are sometimes inferred from common annotated genes/proteins. Such interactions can be mistaken when the gene/protein in question exerts several disparate functions. A case in point is the molecular function of the yeast gene TUM1, an orthologue of the mammalian gene Tst that was proposed as a candidate obesity-resistance gene [7]: we show here that TUM1 and Tst are direct orthologues, yet the mammalian orthologue cannot replace the yeast gene. We also assigned to yeast Tum1 a new function in lipid metabolism, demonstrated by increased SE content in the gene's deletion mutant strain, which is most likely exerted through the regulation of the squalene and/or ergosterol pathway. We demonstrate that while the molecular mechanism for this effect is not known, it is not applied through the tRNA modification pathway, the previously described process to which TUM1 has been annotated. While the evolutionary relationships were clearly established here, the functional relations between yeast TUM1 and mammalian Tst will need to be resolved further.

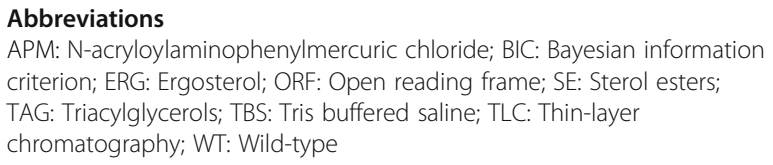

\section{Acknowledgements}

The authors thank Prof. Simon Horvat (National Chemical Institute and University of Ljubljana) for anti-TST antibodies and valuable discussions, Prof. Anders Byström (Umeå University, Sweden) for providing plasmids pABY1653 and pABY525, and Dr. Jernej Šribar (Jožef Stefan Institute) and Gabi Gogg-Fassolter (University Graz) for technical assistance.

\section{Funding}

This work was supported by the grants P1-0207 and N1-0034 of the Slovenian Research Agency and by the Austrian Science Fund, FWF, project I2263-B21 (International Programmes).

\section{Availability of data and materials}

All data generated or analysed during this study are included in this published article.

\section{Authors' contributions}

UP and KN conceived and supervised the study; UP, KN and DK designed experiments; KU, MO and DK performed experiments; KU, KN, DK and UP analysed data; KU, DK and UP wrote the manuscript; KN and MO made manuscript revisions. All authors read and approved the final manuscript.

\section{Ethics approval and consent to participate}

Not applicable.

\section{Consent for publication}

Not applicable.

\section{Competing interests}

The authors declare that they have no competing interests.

\section{Publisher's Note}

Springer Nature remains neutral with regard to jurisdictional claims in published maps and institutional affiliations.

\section{Author details}

1Jožef Stefan Institute, Department of Molecular and Biomedical Sciences, Jamova cesta 39, 1000 Ljubljana, Slovenia. ${ }^{2}$ University of Graz, Institute of Biomolecular Sciences, Humboldtstraße 50/EG, 8010 Graz, Austria. ${ }^{3}$ Biotechnical Faculty, Department of Biology, University of Ljubljana, Večna pot 111, 1000 Ljubljana, Slovenia. ${ }^{4}$ Present address: Institute of Oncology Ljubljana, Zaloška cesta 2, 1000 Ljubljana, Slovenia.

Received: 2 May 2017 Accepted: 10 August 2017

Published online: 22 August 2017

\section{References}

1. Dowhan W. Molecular basis for membrane Phospholipid diversity: why are there so many lipids? Annu Rev Biochem. 1997;66:199-232.

2. Daum G, Lees ND, Bard M, Dickson R. Biochemistry, cell biology and molecular biology of lipids of Saccharomyces cerevisiae. Yeast. 1998;14:1471-510.

3. Souza CM, Pichler H. Lipid requirements for endocytosis in yeast. Biochimi et Biophys Acta. 2007;1771:442-54.

4. Ploier B, Daum G, Petrovič U. Lipid metabolism and Lipidomics. In: Piškur J, Compagno $C$, editors. Molecular mechanisms in yeast carbon metabolism. Berlin, Heidelberg: Springer Berlin Heidelberg; 2014. p. 169-215.

5. Garbarino J, Sturley SL. Saturated with fat: new perspectives on lipotoxicity. Curr Opin Clin Nutr Metab Care. 2009:12:110-6.

6. Klug L, Daum G. Yeast lipid metabolism at a glance. FEMS Yeast Res. 2014; 14:369-88.

7. Morton NM, Beltram J, Carter RN, Michailidou Z, Gorjanc G, McFadden C, et al. Genetic identification of thiosulfate sulfurtransferase as an adipocyteexpressed antidiabetic target in mice selected for leanness. Nat Med. 2016: $771-9$.

8. de Clare M, Oliver SG. Copy-number variation of cancer-gene orthologs is sufficient to induce cancer-like symptoms in Saccharomyces cerevisiae. BMC Biol. 2013;11:24.

9. Noma A, Sakaguchi Y, Suzuki T. Mechanistic characterization of the sulfurrelay system for eukaryotic 2-thiouridine biogenesis at tRNA wobble positions. Nucleic Acids Res. 2009;37:1335-52.

10. Leidel S, Pedrioli PGA, Bucher T, Brost R, Costanzo M, Schmidt A, et al. Ubiquitin-related modifier Urm1 acts as a sulphur carrier in thiolation of eukaryotic transfer RNA. Nature. 2009;458:228-32.

11. Huang B, Lu J, Byström AS. A genome-wide screen identifies genes required for formation of the wobble nucleoside 5-methoxycarbonylmethyl-2thiouridine in Saccharomyces cerevisiae. RNA. 2008;14:2183-94.

12. Saccharomyces Genome Database. Stanford University, Stanford. 2017. http://yeastgenome.org. Accessed 19 July 2017.

13. National Center for Biotechnology Information. US National Library of Medicine, Rockville. 2017. http://www.ncbi.nlm.nih.gov. Accessed 19 July 2017.

14. Gertz EM, Yu Y-K, Agarwala R, Schäffer AA, Altschul SF. Composition-based statistics and translated nucleotide searches: improving the TBLASTN module of BLAST. BMC Biol. 2006:4:41.

15. Bordo D, Bork P. The rhodanese/Cdc25 phosphatase superfamily. Sequencestructure-function relations EMBO Rep. 2002;3:741-6.

16. Cipollone R, Ascenzi P, Visca P. Common themes and variations in the rhodanese superfamily. IUBMB Life 2007:59:51-9.

17. ExPASy Bioinformatics Resource Portal. Swiss Institute of Bioinformatics, Lausanne. 2017. Accessed 19 July 2017.

18. Simple Modular Architecture Research Tool. http://smart.embl-heidelberg.de Accessed 19 July 2017

19. InterPro Protein sequence analysis \& classification. EMBL-EBI, Wellcome Genome Campus, Hinxton. 2017. http://www.ebi.ac.uk/interpro. Accessed 19 July 2017

20. The Pfam protein families database. EMBL-EBI, Wellcome Genome Campus, Hinxton. 2017. http://pfam.janelia.org/. Accessed 19 July 2017.

21. Sievers F, Wilm A, Dineen D, Gibson TJ, Karplus K, Li W, et al. Fast, scalable generation of high-quality protein multiple sequence alignments using Clustal omega. Mol Syst Biol. 2011;7:539. 
22. Trifinopoulos J, Nguyen LT, von Haeseler A, Minh BQ. W-IQ-TREE: a fast online phylogenetic tool for maximum likelihood analysis. Nucleic Acids Res. 2016:44:232-5.

23. Guindon S, Dufayard JF, Lefort V, Anisimova M, Hordijk W, Gascuel O. New algorithms and methods to estimate maximum-likelihood phylogenies: assessing the performance of PhyML 3.0. Syst Biol. 2010;59:307-21.

24. Saitou N, Nei M. The neighbor-joining method: a new method for reconstructing Phylogenetic trees. Mol Biol Evol. 1987;4:406-25.

25. Ensembl. EMBL-EBI, Wellcome Genome Campus, Hinxton. 2017. http://www. ensembl.org/index.html. Accessed 19 July 2017.

26. Entrez Gene. National Center for biotechnology information. Rockville: US National Library of Medicine; 2017. http://www.ncbi.nlm.nih.gov/gene. Accessed 19 July 2017

27. Muffato M, Louis A, Poisnel CE, Crollius HR. Genomicus: a database and a browser to study gene synteny in modern and ancestral genomes. Bioinformatics. 2010;26:1119-21.

28. Niedenthal RK, Riles L, Johnston M, Hegemann JH. Green fluorescent protein as a marker for gene expression and subcellular localization in budding yeast. Yeast. 1996;12:773-86.

29. Lu J, Huang B, Esberg A, Johansson MJO, Byström AS. The Kluyveromyces lactis gamma-toxin targets tRNA anticodons. RNA. 2005;11:1648-54.

30. De Crécy-Lagard V, Brochier-Armanet C, Urbonaviius J, Fernandez B, Phillips $G$, Lyons B, et al. Biosynthesis of wyosine derivatives in tRNA: an ancient and highly diverse pathway in Archaea. Mol Biol Evol. 2010;27:2062-77.

31. Kurat CF, Wolinski H, Petschnigg J, Kaluarachchi S, Andrews B, Natter K, et al. Cdk1/Cdc28-dependent activation of the major Triacylglycerol lipase Tgl4 in yeast links Lipolysis to cell-cycle progression. Mol Cell. 2009;33:53-63.

32. Hanscho M, Ruckerbauer DE, Chauhan N, Hofbauer HF, Krahulec S, Nidetzky $\mathrm{B}$, et al. Nutritional requirements of the BY series of Saccharomyces cerevisiae strains for optimum growth. FEMS Yeast Res. 2012;12:796-808.

33. Gough J, Karplus K, Hughey R, Chothia C. Assignment of homology to genome sequences using a library of hidden Markov models that represent all proteins of known structure. J Mol Biol. 2001:313:903-19.

34. Parfrey LW, Lahr DJG, Knoll AH, Katz LA. Estimating the timing of early eukaryotic diversification with multigene molecular clocks. Proc Natl Acad Sci U S A. 2011;108:13624-9.

35. Kachroo AH, Laurent JM, Yellman CM, Meyer AG, Wilke CO, Marcotte EM Evolution. Systematic humanization of yeast genes reveals conserved functions and genetic modularity. Science. 2015;348:921-5.

\section{Submit your next manuscript to BioMed Central and we will help you at every step:}

- We accept pre-submission inquiries

- Our selector tool helps you to find the most relevant journal

- We provide round the clock customer support

- Convenient online submission

- Thorough peer review

- Inclusion in PubMed and all major indexing services

- Maximum visibility for your research

Submit your manuscript at www.biomedcentral.com/submit

) Biomed Central 